OPTIMAL TERMINATION AND THE IRR REVISITED

by

David Robinson

Assistant Professor

Department of Economics

Laurentian University

$\&$

W. Rupert Cook Ph.D

Professor

Department of Economics

Laurentian University

On leave in Department of Economics

University of Western Australia 1990-1991

DISCUSSION PAPER 91.08

JUY 1991 


\section{OPTIMAL TERMINATION AND THE IRR REVISITED}

\section{Introduction}

For more than fifty years, a star studded cast of economists has been involved in a minor, off-broadway production. Keynes, Hicks, Arrow, and Sen are among the best known leading players, but many others have played major roles1. "The Analysis of the Internal Rate of Return on a Capital Project" is still running, and will still provide an evening's edification and entertainment. Dramatic tension centers on two questions: Will the Internal Rate of Return be unique, allowing it to claim a role in project selection; and will the Marginal Efficiency of Capital Schedule slope down, so that it can hold up one end of the Keynesian I-S curve? It gives nothing away to say that the plot revolves around the decisions of a rational economic agent.

This paper presents an updated version of the story. To make the plot more relevant to a modern audience, our protagonist is more like the common man or woman: she may not always choose optimally, but her decisions are consistent with her interests in a weaker sense. She is less predictable than the strictly maximizing heroine of earlier versions, but she is not too perverse. The language has been simplified as well, in order to bring the economic motives of the main character to the surface, and we pay more attention to the relationships between characters than previous authors have.

Cannaday, Colwell and Paley provide a survey of the literature. We need only remind readers that, building on earlier work, Arrow and Levhari 
(1969) showed that for a special class of projects, choosing the project life, T, to maximize the present value of a project is sufficient to ensure that the present value is a monotonically decreasing function of the discount rate. It follows that for such projects the IRR is unique if it exists at all. J.S. Flemming and J.F. Wright generalized the Arrow and Levhari results to the discrete case with variable discount rates. D.M. Nuti (1973), using an argument developed by J.F. Wright (1959), provided an elegant simple proof for the discrete case. Sen (1975) produced the minimal sufficient conditions for the discrete with variable discount rates and a stream of "consequences" from shutting down that extends both before and after the nominal termination time.

We present the minimal sufficient conditions for the continuous case. We show, using a more direct argument, that Sen's minimal conditions are more complex than necessary, and not, in general, sufficient. Our conditions are economically meaningful and apply to a larger class of projects. We also argue that monotonicity of the capital value of a project, and non-uniqueness of the IRR are linked to unavoidable conflicts of interest, and should be understood as a signal that there may be important problems to resolve.

In the first section below we demonstrate that the sufficient condition for a unique internal rate of return in the standard version of the problem is not optimality but rather a condition we call "incentive consistency", combined with another weak consistency requirement that we call "a lack of barn door perversity". Incentive consistency is related to the concept of incentive compatibility in the game theory literature, in which a mechanism is incentive compatible if telling the truth is a Nash equilibrium. An announced terminal time is incentive consistent if there is no earlier truncation that yields a higher project value. It would be incentive compatible if the project owners could not make themselves better off by truncating earlier. The relationship between the two concepts will be explored below.

In the second part of the paper we show in a more general model that even choosing project life ex ante to maximize the present value of the project does not guarantee that the IRR is unique. If, however, the shut-down date is incentive consistent in the more general model, then the IRR will still be unique. In the concluding section we briefly consider the implications of incentive consistency for the evaluation of certain public projects and for the form of certain contracts.

\section{Part 1: The role of incentive consistency in the simple model}

It is convenient to define the following terms. The present value of a project, computed at time $t$, is $V(r, T, t)$, where $r$ is the discount rate 2 and $T$ is the lifetime of the project. The flow of net benefits at any time $t$ is $x(\tau, T)$, and

$$
V(r, T, t) \equiv \int_{t}^{T} e^{-r \tau} x(\tau, T) d \tau
$$

The expected life of a project will in general affect the stream of net benefits, $x(\tau, T)$, through the choice of capital stock, investment timing, and even marketing schedules. Previous authors have implicitly assumed that the stream of net benefits is insensitive to the length of the project 3 .

2. Extending our analysis to the case with a discount rate that is a function of time is a simple exercise which we leave to the reader. We have chosen to focus on the case with a uniform discount rate because of the connection with the IRR.

3 Although Sen assumes that the stream of net benefits is independent of $\mathrm{T}$, he adds a stream of shutdown costs which does depend on $T$. His model is analogous to ours if the consequent stream is incorporated in $\mathrm{x}$ and the end of the sequence stream is defined as the end of the project. 
We are concerned with the properties of $\mathrm{V}$ when $\mathrm{T}$ is chosen by rational economic agents, so we define:

Optimality: A finite project lifetime $\mathrm{T}^{*}$ is optimal if

$$
\mathrm{V}\left(\mathrm{r}, \mathrm{T}^{*}, 0\right) \geq \mathrm{V}(\mathrm{r}, \mathrm{T}, 0) \quad \text { for all } \mathrm{T}
$$

The optimal lifetime for the project is chosen before the project is undertaken. The rational economic agent, however, is aware of a radical, almost existential, freedom to alter her plans even after the project is under way; if she should discover that she suffers a loss in proceeding to the originally planned terminal time, she would truncate the project at a time different from that originally chosen. Clearly we are suggesting a kind of time-inconsistency that may make the return to planning-to-complete different from the return to actually completing a project. Such a situation is familiar from the literature on commitment and the time consistency of public policies. We therefore define

Incentive Consistency: A finite project lifetime $\mathrm{T}^{*}$ is incentive consistent if, for every $\mathrm{t}<\mathrm{T}^{*}$, continuing to $\mathrm{T}^{*}$ does not decrease the realized capital value of the project 4 . More formally,

$$
\mathrm{V}\left(\mathrm{r}, \mathrm{T}^{*}, \mathrm{t}\right) \geq 0 \quad \text { for all } \mathrm{t}<\mathrm{T}^{*}
$$

In the absence of real opportunity costs, incentive consistency implies that if a rational agent were free to change her plans at any time, having chosen $\mathrm{T}^{*} \mathrm{ex}$ ante, she would not truncate the project before $\mathrm{T}^{*}$. The absence of opportunity costs is crucial. Consider two projects with the same sequence of costs and revenues. One, a highway project, involves a planned commitment of real capital at time $t$ to expand the right of way. There are alternative uses

4 Incentive consistency appears in Sen's analysis as "preoptimality" and as two
conditions on the "consequence stream". He defines preoptimality indirectly: $T^{*}$ is Tother conditions on the "consequence stream". He defines preoptimality indirectly: $T^{*}$ is $T$ -
optimal if $V\left(r, T^{*}, 0\right)=V(r, t, 0)$ for all $t \leq T, T^{*}$ is preoptimal. The other conditions are optimal if $\mathrm{V}\left(\mathrm{r}, \mathrm{T}^{*}, \mathrm{O}\right.$
discussed below. for the capital, so that the return to proceeding with the expansion must not only be greater than zero $[\mathrm{V}(\mathrm{r}, \mathrm{t}, \mathrm{T})>0\}$, but it must also dominate other feasible uses of the capital. The other project is a manufacturing process, it requires an expenditure on inputs at time $t$, but since the inputs may be purchased on credit from the suppliers, and there is no altemative use for that credit, there is no real opportunity costs involved. Incentive consistency in this case is equivalent to incentive compatibility.

Incentive consistency neither implies nor is implied by optimality. The project life that maximizes $V$ ex ante may be preceded by a sequence of times which yield successively higher local maxima of $V$, each greater than zero. Each may be incentive consistent, and each is sup-optimal. We will show in section 2 that optimality does not imply incentive consistency in general.

Incentive consistency implies that what we term "the order condition" is satisfied for any interval $\left[\mathrm{t}<\mathrm{T}^{*}, \mathrm{~T}^{*}\right]$ :

The Order Condition for $r$ : A net benefit function $x(t, T)$ satisfies the order condition on an interval $\left[t_{i} t_{2}\right]$ at a discount rate $r$ when for every sub-interval in $\left[t_{1} t_{2}\right]$ that contributes negative present value to $V$, there is a later sub-interval which contributes an equal or greater positive bit of present value at the given $r$.

To see that the order condition must be satisfied for the entire life of the project if the end of the project, $\mathrm{T}^{*}$, is incentive consistent, consider any $\mathrm{t}_{\mathrm{i}}<$ $T^{*}$ which is immediately followed by an interval ending at $t_{2}$ for which the present value is negative. Since the present value on that interval is a negative quantity, say -c, incentive consistency implies that the present value on the interval $\left[t_{2}, T^{*}\right]$ is positive and at least as large as $c$. The order condition is 
therefore satisfied for $\left[t_{1}, T^{*}\right]$, and for every $t \in\left[0, T^{*}\right]$. The converse is also true: if the stream of net benefits from a project satisfies the order condition on the interval $\left[0, T^{*}\right]$ for the discount rate $\mathrm{r}$, then $\mathrm{T}^{*}$ is incentive consistent.

It is the order condition that does the work in the following arguments. It is a purely technical condition, however. Incentive consistency, on the other hand, is concerned with economic behaviour. Since incentive consistency is both necessary and sufficient for the order condition to be satisfied we need consider only the implications of the order condition.

PROPOSITION 1: If the order condition is satisfied for $\mathbf{r}$, and the undiscounted stream of net benefits is independent of $r$ and $T$, then the present discounted value of the project is decreasing in $r$.

PROOF: The order condition guarantees that negative elements are matched by later positive elements. Time constancy of $x(\tau, T)$ ensures that changing $r$ does not affect the pattern of net benefits. Let the ratio of discount factors at $\mathrm{t}_{1}$ and $\mathrm{t}_{2}, \mathrm{t}_{\mathrm{i}}<\mathrm{t}_{2}$, be:

$$
R(r)=\left\{e^{-r_{2}} / e^{-r t_{1}}\right\}
$$

Since $\partial R / \partial r=\left(t_{1}-t_{2}\right) e^{r\left(t_{1}-t_{2}\right)}<0, \quad$ it follows that if $r$ rises, the discount factor will increase more for the later positive elements than for negative elements that precede them. The order condition guarantees that early negative elements are matched by later positive elements and therefore the present value of the entire project falls.

PROPOSITION 2: If the order condition is satisfied $\mathbf{r} \geq \mathbf{0}$ for a fixed $T$, the undiscounted stream of net benefits is initially negative, independent of $r$ and $T$, bounded in magnitude and

\section{bounded in duration, then the IRR exists and is unique.}

Since the capital value must fall as $r$ rises, there can only be one value of $x$ for which the capital value of a project is zero. If the order condition is satisfied for $r \geq 0$, the capital value of the project is non-negative without discounting. Clearly the ratio of discount factors defined in the previous paragraph can be made arbitrarily small, so that as long as the benefit stream is bounded and finite, it is possible to choose $r$ sufficiently large to ensure that the IRR exists.

Propositions 1 and 2 show that incentive consistency is sufficient to guarantee monotonicity and a unique IRR if the terminal time is fixed. As $\mathrm{r}$ changes, however, the terminal time chosen might also change. One solution is to consider only cases where the terminal point is chosen optimally. It is obvious that, when $x(\tau, T)$, is independent of $T$, that every optimal terminal point is both incentive consistent, and yields the same project value. It follows that the project value falls when $r$ rises even if the optimal terminal point changes, either continuously or discretely.

Since incentive consistency does the work in any demonstration that optimal termination ensures a unique IRR, it is natural to wonder whether we can dispense with optimality. Since incentive consistency is a weaker condition than optimality, a proof using only incentive consistency expands the set of projects for which the IRR is known to be unique. It turns out than an additional, very weak, consistency assumption is needed. It is easy to see that a move to any earlier incentive consistent $\mathrm{T}$ in response to a rise in $\mathrm{r}$ must be accompanied by a fall in the capital value of the project. Now consider a case for which $T^{* *}>T^{*}$ is also incentive consistent. The only complication arises 
if the agent who chose $\mathrm{T}^{*}$ at the initial $\mathrm{r}$ suddenly chooses $\mathrm{T}^{* *}$ when $\mathrm{r}$ rises5. Clearly, if,

$$
\mathrm{V}\left(\mathrm{r}, \mathrm{T}^{* *}, 0\right)=\mathrm{V}\left(\mathrm{r}, \mathrm{T}^{*}, 0\right)
$$

such a decision

would be irrational since the value of extending the life of the project at any rate of interest higher than $r$ is negative. If $V\left(r, T^{* *}, 0\right)$ is larger than $V(r$, $T^{*}, 0$ ), such a decision would be rational but perverse. Rational because extending the project life increases its value, and perverse because such an extension would have been even better had the decision been made before the interest rate rose. Extending the project when the interest rate rises is rather like closing the barn door after some of the horses have escaped. If we rule out what might be termed "barn door perversity", we have the following sufficiency result:

Incentive consistency is sufficient to guarantee monotonicity and a unique IRR if the terminal time is fixed, but much of the interest lies in the possibility that the choice of the terminal time might depend on $\mathrm{r}$.

PROPOSITION 3: If the life of a project is always incentive consistent, and if agents do not exhibit barn door perversity, then the present value of the project is monotonically declining in the discount rate and the Internal Rate of Return is unique if it exists.

The function of the optimality assumption in previous proofs is to ensure that the condition we call incentive consistency is satisfied. Optimality itself is not required, and as we shall show, does not always guarantee that incentive consistency is satisfied. Incentive consistency, on the other hand, is both necessary and sufficient for the order condition to hold, and the order condition guarantees monotonicity. Optimality is considerably more

$5 \quad$ Much of the difficulty of the proof by Arrow and Levhari arises because of the need to rule out such shifts. restrictive than the combination of incentive consistency and a lack of barn door perversity, and hence the argument given here is not only easier than those of previous authors, but more general. It seems reasonable to assume that projects undertaken by private agents on their own behalf will be incentive consistent. Problems tend to be confined to cases where an agent undertakes a project on behalf of another. We will argue later that most projects in the public sector will also be incentive consistent from the point of view of the public agency, and that most observed private contracts will also be incentive consistent. Since incentive consistency is generally less restrictive than optimal termination, the set of projects for which the internal rate of return is unique is clearly larger than previously thought.

\section{PART 2: INCENTIVE CONSISTENCY AND OPTIMALITY}

The relationship between incentive consistency and optimality is clearer in a model that is slightly richer than previous authors have considered. We therefore consider an extended version of the model analyzed by Arrow and Levhari.

As in the previous section let $\mathrm{x}(\tau, \mathrm{T})$, be a known, continuous 6 stream of net income. Let $g(t, T)$ be the scrap value or instantaneous shut-down cost if the project, originally planned to end at $T$, is terminated at $t$. Previous proofs restrict $g(t, T)$ to positive values7. Notice that if there are costs involved in preparing for the shut-down they can be incorporated in $x(t, T)$.

6 Continuity is not necessary, as Flemming and Wright (1971) demonstrates. The argument presented below is easily adapted to to the discrete case

7 Sen (1975) allows for an entire stream of "consequences" that may continue after the nominal shut down date. His set of minimal sufficiency conditions for monotonicity include two separate restrictions(non-negative consequence sums and non-negative consequence remainders) that are equivalent to imposing incentive consistency over the entire stream of benefits and costs. 
The present value of the project at a constant 8 discount rate $r$, when evaluated at $t$, is

(1)

$$
\begin{aligned}
V(r, T, t) & =\int_{0}^{T} e^{-r t} x(t, T) d t+e^{-r T} g(T, T) \\
& =\int_{0}^{T} e^{-r t} x(t, T) d t+\int_{0}^{T} e^{-r t}\left\{g_{t}(t, T)-r g(t, T\} d t+g^{*}\right.
\end{aligned}
$$

where $\mathrm{g}^{*}$ is the scrap value or shut-down cost at the moment after the project is begun. The function $g_{t}(t, T)$ is the rate of change of the scrap value through time and $\mathrm{rg}(\mathrm{t}, \mathrm{T})$ is the interest lost if realisation of the scrap value is postponed. Equation (i) is equivalent to

$$
V(r, T, t)=\int_{0}^{T} e^{-r t} X(t, T, r) d t+g^{*}
$$

where $X(t, T, r)=x(t, T)+g_{t}(t, T)-r g(t, T)$. It is $X$, and not its components, that is economically significant 9 .

$$
\text { If a finite terminal point } T^{*} \text { maximizes } V \text {, }
$$

$$
\mathrm{V}\left(\mathrm{r}, \mathrm{T}^{*}, 0\right) \geq \mathrm{V}(\mathrm{r}, \mathrm{t}, \mathrm{0}) \quad \text { for all } \mathrm{t}
$$

Equation (2) holds for all $t$ less than $\mathrm{T}^{*}$, hence:

$8 \quad$ Flemming and Wright consider the case of a discount rate which is variable but independent of the project.

9 Arrow\&Levhari, Flemming\&Wright and others first consider the net direct revenue function, $x$, then, to preserve their result when the scrap function is introduced, they impose separate conditions on $\mathrm{g}$,

$$
0 \leq \int_{T=t}^{\mathrm{T} *} \mathrm{~V}_{\mathrm{T}}(\mathrm{r}, \mathrm{T}, 0) \mathrm{dT}
$$

which implies:

$0 \leq \int_{T=t}^{T *} \int_{\tau=0}^{t} e^{-r \tau} X_{T}(\tau, T, r) d \tau d T$

$0 \leq \int_{\mathrm{T}=\mathrm{t}}^{\mathrm{T} *} \int_{\tau=0}^{\mathrm{t}} \mathrm{e}^{-\mathrm{r \tau}} \mathrm{X}_{\mathrm{T}}(\tau, \mathrm{T}, \mathrm{r}) \mathrm{d} \tau \mathrm{dT}+\int_{\tau=\tau}^{\mathrm{T} *} \mathrm{e}^{-\mathrm{rt}} \mathrm{X}\left(\tau, \mathrm{T}^{*}, \mathrm{r}\right) \mathrm{d} \tau$

$0 \leq \int_{\mathrm{T}=\tau \tau=0}^{\mathrm{T}} \int_{\mathrm{T}}^{\mathrm{t}} \mathrm{e}^{-\mathrm{rt}} \mathrm{X}_{\mathrm{T}}(\tau, \mathrm{T}, \mathrm{r}) \mathrm{d} \tau \mathrm{dT}+\mathrm{e}^{-\mathrm{rt}} \mathrm{V}\left(\mathrm{r}, \mathrm{T}^{*}, \mathrm{t}\right)$

In the simple case considered by previous authors, the stream of net benefits is independent of the date at which the project is ended. $X_{T}=0$ in that case, and the first term vanishes. The remaining term is simply the present value of the interval from $t$ to $T^{*}$, evaluated at $t$ and discounted to the beginning of the project. Optimality implies incentive consistency when the double integral is non-positive. It follows that

PROPOSITION 4: The internal rate of return is unique when the project lifetime has been optimally chosen and the stream of net benefits is independent of the time at which the project is terminated. 


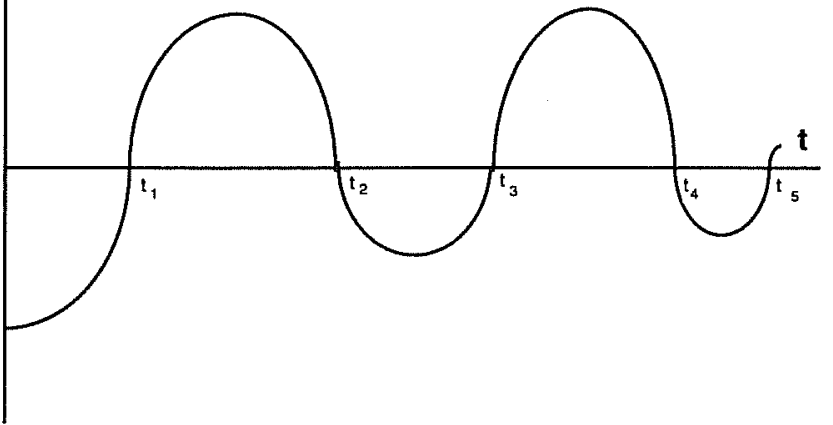

Figure 1. Net Benefit as a Function of $t$

For most projects the revenue stream will vary as the project life changes. When $X_{T}$ is non-zero, extending the project life from $t$ to $T^{*}$ affects the present value generated in the interval $(0, t)$. Since the first term may be positive, the second may be negative, as figure 1 illustrates. An interesting example might occur if there were a body of ore that could be extracted either by sub-surface mining, or by the cheaper open-pit method. Assume the production period is unaffected, but the open-pit method requires a protracted period of restoration after production ends, and so involves a later terminal time. The longer project is optimal. The second term above is thus negative, and a rational agent would truncate the project early to avoid the negative returns in the final periods. In this case $\mathrm{T}^{*}$ is optimal ex ante, but not incentive consistent. It is a simple exercise to choose $X\left(\tau, T^{*}, r\right)$ so that the internal rate of return is not unique. Hence:

PROPOSITION 5: Optimal choice of the project life is not sufficient to ensure that the internal rate of return is unique if the stream of net benefits is not independent of the lifetime of the project.

\section{PART 3: CONCLUDING REMARKS}

A unique IRR is assured whenever the project's "internal" incentives are sufficient to induce a free agent to continue at least to the end of the project. "Internal" incentives include all costs and benefits arising from the project up to and including the intended termination, including scrap value and any shut-down costs. We emphasise that the relevant terminal date is the date of the last cost or benefit received and not simply the nominal project end, as in Sen's analysis. If there is a stream of consequence which accrie after the nominal project end, they bear on the monotonicity of capital value (as Sen showed) exactly as much as those accruing before the nominal project ending.

The "internal" incentives are entirely determined by the net benefit function, $X(\tau, T, r)$, but behaviour may be affected by opportunities outside the project. If resources can be withdrawn from a later stage of the project and applied to another project with a higher return, a rational agent might truncate the project early despite internal incentive consistency. The example of a highway project with a planned extension was given above. Such a project is separable, in the sense that it is really two separate projects, each with its own internal rate of return. If we observe that the second stage is undertaken, we know it has satisfied a condition which is stronger than mere incentive consistency.

The concept of incentive consistency can cast some light on another problem in the project evaluation literature. Consider the case of an open-pit mine, cited by Sugden and Williams (1978), or Ross, Spatt and Dybvig (1980). The open pit technique may be the one which minimizes both private and social costs, but it involves expenditures after production ends. Multiple internal rates of return may result. Notice, however, that continuing until the pit has been filled is not incentive consistent for an agent who cannot capture 
the amenities that filling the pit would produce. A private operator would prefer to close the mine and leave the pit to be filled in by others. In the absence of binding contracts, defection would be the rule. Thus the stream of benefits that causes multiple internal rates of return is associated with an enforcement problem.

In practice, there are mechanisms for enforcing such contracts and there are contract forms that are self enforcing. In the above example, the cost of filling in the pit can be made unavoidable by requiring the operator to post a bond in advance. the cost of filling in the pit is then incurred, for all practical purposes, in the first moment of the project ${ }^{10}$. A contractual arrangement that resolves the conflict between the public interest and that of the mining company also eliminates the source of multiple internal rates of return 11 .

By focusing on the concept of incentive consistency we have been able to clarify the conditions under which the capital value of a project is a monotonic declining function of the discount rate. We have also shown, contradicting earlier writers, that the optimal choice of the project lifetime does not guarantee monotonicity. Our approach is very much in the spirit of the earlier literature, however, we show that projects undertaken by reasonably sensible economic agents will have nice properties. Our main

10 Mishan proposed that a sinking fund be established for public projects with shutdown costs. The effect is similar, although he was apparently looking for an accounting procedure that would make the problem of multiple IRRs disappear, and not trying to employ appear so ad hoc, can be given a sound economic rationalisation.

11 This conclusion appears to contradict Sen's assertion that "The more the government passes on the costs of environmental damage to private firms through taxes and other charges, the less likely it is that (the conditions for monotonicity) must be fulfilled in private project evaluation." contribution lies in showing that less than full optimizing behaviour is sufficient 12 , thereby expanding the set of well-behaved projects.

\section{Bibliography}

is

Arrow, Kenneth J., and Levhari, David,; "Uniqueness of the Internal Rate of Return with Variable Life of Investment"; Economic Journal Sept (1969) 560-66.

Cannaday,Roger E., Peter F Colwell and Hiram Paley; "Relevant and Irrelevant Internal Rates of Return", The Engineering Economist Fall (1986) 17-38

Flemming, J.S., and Wright. J. F, "Uniqueness of the Internal Rate of Return: A Generalisation"; The Economic Joumal, June (1971) 256-64.

Mishan, E.J. Cost-benefit analvsis : an informal introduction Rev.New Edn. London 1975

Nuti, D.M., "On the Truncation of Production Flows"; Kyklos: 26 (1973) 485-495.

Ross, Stephen A., Chester S. Splatt, Philip H Dybvig; "Present Value and Internal Rate of Return"; Journal of Economic Theory 23,(1980) 66-81.

Sen, Amartya K., "Minimal Conditions for Moinotonicity of Capital Value"; Journal of Economic Theorv: 11 (1975) 340-355.

Silcock, T.H., "Complementarity and Future Time: A note on the Marginal Efficiency of Capital", The Economic Journal Dec. (1959) 816-19.

Sugden, Robert, and Alan Williams; The Principles of Cost Benefit Analysis O.U.P, Oxford (1978).

Wright, J.F.; "The Marginal Efficiency of Capital", The Economic Journal Dec. (1959) 813 16.

12 We stress that previous authors have made use of the property we call incentive consistency. For most, however it was simply a consequence of optimality. Sen's minimal sufficiency conditions include incentive consistency, but under three separate names. 